\title{
CHERN-SCHWARTZ-MACPHERSON CLASSES FOR SCHUBERT CELLS IN FLAG MANIFOLDS
}

\author{
PAOLO ALUFFI AND LEONARDO C. MIHALCEA
}

\begin{abstract}
We obtain an algorithm computing the Chern-Schwartz-MacPherson (CSM) classes of Schubert cells in a generalized flag manifold $G / B$. In analogy to how the ordinary divided difference operators act on Schubert classes, each CSM class of a Schubert class is obtained by applying certain Demazure-Lusztig type operators to the CSM class of a cell of dimension one less. These operators define a representation of the Weyl group on the homology of $G / B$. By functoriality, we deduce algorithmic expressions for CSM classes of Schubert cells in any flag manifold $G / P$. We conjecture that the CSM classes of Schubert cells are an effective combination of (homology) Schubert classes, and prove that this is the case in several classes of examples. We also extend our results and conjectures to the torus equivariant setting.
\end{abstract}

\section{INTRODUCTION}

A classical problem in Algebraic Geometry is to define characteristic classes of singular algebraic varieties generalizing the notion of the total Chern class of the tangent bundle of a non-singular variety. The existence of a functorial theory of Chern classes for possibly singular varieties was conjectured by Grothendieck and Deligne, and established by R. MacPherson [Mac74]. This theory associates a class $c_{*}(\varphi) \in H_{*}(X)$ with every constructible function $\varphi$ on $X$, such that $c_{*}\left(\mathbb{1}_{X}\right)=c(T X) \cap[X]$ if $X$ is a smooth compact complex variety. (The theory was later extended to arbitrary algebraically closed fields of characteristic 0, with values in the Chow group [Ken90], [Alu06b].) The strong functoriality properties satisfied by these classes determine them uniquely; we refer to 93 below for details. If $X$ is a compact complex variety, then the class $c_{*}\left(\mathbb{1}_{X}\right)$ coincides with a class defined earlier by M. H. Schwartz [Sch65a, Sch65b] independently of the work mentioned above. This class is commonly known as the Chern-Schwartz-MacPherson (CSM) class of $X$. In general, we denote by $c_{\mathrm{SM}}(W)$ the class $c_{*}\left(\mathbb{1}_{W}\right) \in H_{*}(X)$ for any constructible (e.g., locally closed) subset $W \subseteq X$.

Let $G$ be a complex simple Lie group and let $B$ be a Borel subgroup. Denote by $W$ the Weyl group of $G$. The goal of this note is to provide an algorithm calculating the CSM classes of the Schubert cells $X(w)^{\circ}:=B w B / B$ in the generalized flag manifold $G / B$, as $w$ varies in $W$. To describe the answer, we need to recall two well known families of operators on the homology group $H_{*}(G / B)$; we refer to $\$ 2$ below for details.

Let $s_{1}, \ldots, s_{r} \in W$ be the simple reflections corresponding respectively to the simple roots $\alpha_{1}, \ldots, \alpha_{r}$ of $G$, and let $\ell: W \rightarrow \mathbb{N}$ be the length function. Denote by $X(w):=\overline{B w B / B}$ the Schubert variety corresponding to $w$; it is a subvariety of $G / B$ of complex dimension $\ell(w)$. For each $1 \leqslant k \leqslant r$, the classical BGG operator BGG73] is an operator $\partial_{k}: H_{*}(G / B) \rightarrow$ $H_{*+2}(G / B)$ which sends the Schubert class $[X(w)]$ to $\left[X\left(w s_{k}\right)\right]$ if $\ell\left(w s_{k}\right)>\ell(w)$ and to 0

P. A. was supported in part by a Simons Collaboration Grant and NSA Award H98230-15-1-0027; L. C. M. was supported in part by a Simons Collaboration Grant and by NSA Young Investigator Award H98230-13$1-0208$. 
otherwise. The Weyl group admits a right action on $H_{*}(G / B)$, which was originally used to define the BGG operator $\partial_{k}$. For $1 \leqslant k \leqslant r$ define the non-homogeneous operator

$$
\mathcal{T}_{k}:=\partial_{k}-s_{k}: H_{*}(G / B) \rightarrow H_{*}(G / B),
$$

where $s_{k}$ denotes the (right) action of the simple reflection $s_{k}$. The main result of this note is the following.

Theorem 1.1. Let $w \in W$ be a Weyl group element and $1 \leqslant k \leqslant r$. Then

$$
\mathcal{T}_{k}\left(c_{S M}\left(X(w)^{\circ}\right)\right)=c_{S M}\left(X\left(w s_{k}\right)^{\circ}\right) .
$$

In the case $w=\mathrm{id}$, the Schubert cell $X(\mathrm{id})^{\circ}$ is a point, and $c_{\mathrm{SM}}([p t])=[p t]$. More generally, if $w=s_{i_{1}} \ldots s_{i_{k}}$, then the theorem implies that the CSM class $c_{\mathrm{SM}}\left(X(w)^{\circ}\right)$ is obtained by composing the operators $\mathcal{T}_{i_{k}} \cdots \mathcal{T}_{i_{1}}$. This is reminiscent of the classical situation in Schubert Calculus, where one generates all the Schubert classes by applying successively the BGG operators $\partial_{k}$. To further the analogy, $\partial_{k}^{2}=0$ and the BGG operators satisfy the braid relations. We prove that $\mathcal{T}_{k}^{2}=1$, and then Theorem 1.1 can be used to show that the operators $\mathcal{T}_{k}$ also satisfy the braid relations (Proposition 4.1). In particular, there is a well defined operator $\mathcal{T}_{w}$ associated with any $w \in W$, and this yields a new representation of the Weyl group on $H_{*}(G / B)$. The CSM classes of the Schubert cells $X(w)^{\circ}$ are the values obtained by applying to the class of a point $[p t]$ the operators $T_{w^{-1}}$ in this representation. Further, the action of each simple reflection $\mathcal{T}_{k}$ on Schubert classes can be written explicitly using the Chevalley formula (Proposition 4.3). This gives an explicit algorithm to calculate the CSM class of any Schubert cell.

We also note that $\mathcal{T}_{k}$ is related to a specialization of the Demazure-Lusztig operator defined in Gin98, §12], in relation to degenerate Hecke algebras. We plan on investigating this connection further in a future paper.

Perhaps the most surprising feature of the CSM classes (and of the operators $\mathcal{T}_{k}$ ) is a positivity property. It follows from the definition of CSM classes that

$$
c_{\mathrm{SM}}\left(X(w)^{\circ}\right)=\sum_{v \leqslant w} c(v ; w)[X(v)]
$$

where $\leqslant$ denotes the Bruhat ordering, and $c(w ; w)=c(\mathrm{id} ; w)=1$. Despite the fact that $\mathcal{T}_{k}$ does not preserve the positivity of a combination of Schubert classes, we conjecture that $c(v ; w)>0$ for all $v \leqslant w$. We have checked this by explicit computations for thousands of Schubert cells $X(w)$ in type A flag manifolds $\operatorname{Fl}(n)$ for $n \leqslant 8$. We were also able to prove that this positivity holds for several families of Schubert cells, across all Lie types; see $\$ 5$ below.

Let $P \subset G$ be a parabolic subgroup containing $B$, and let $p: G / B \rightarrow G / P$ be the natural projection. Then $p\left(X(w)^{\circ}\right)=X\left(w W_{P}\right)^{\circ}$, where $W_{P}$ is the subgroup of $W$ generated by the reflections in $P$. The functoriality of CSM classes can be used to prove that

$$
p_{*}\left(c_{\mathrm{SM}}\left(X(w)^{\circ}\right)\right)=c_{\mathrm{SM}}\left(X\left(w W_{P}\right)^{\circ}\right)
$$

(see Proposition 3.5 below). In particular, the (conjectured) positivity of CSM classes of Schubert cells in $G / B$ implies the positivity of classes in any $G / P$. In the case when $G / P$ is a Grassmann manifold this was proved in several cases by the authors of this note [AM09, Mih15], B. Jones [Jon10], J. Stryker [Str11], and it was settled for all cases by J. Huh [Huh]. Huh was able to realize the homogeneous components of CSM classes as a class of an effective cycle, but unfortunately his main technical requirements do not seem to hold for arbitrary flag manifolds $G / B$. 
Our calculation of CSM classes is based on a construction of these classes in terms of bundles of logarithmic tangent fields. For every $W \subseteq X$, the class $c_{\mathrm{SM}}\left(\mathbb{1}_{W}\right) \in H_{*}(X)$ may be obtained by pushing forward to $X$ the total Chern class of $T_{\widetilde{W}}(-\log D)$, where $\widetilde{W}$ is a resolution of $\bar{W}$ such that $D:=\widetilde{W} \backslash W$ is a simple normal crossing (SNC) divisor (Alu99, Alu06a]). We refer to 93 for details. This approach was used successfully in previous work by the authors [AM09] to compute CSM classes of Schubert cells in the Grassmann manifold and prove partial positivity results. In that case one can use a resolution of a Schubert variety which is a (smooth) Schubert variety, but in a partial flag manifold. B. Jones [Jon10] gave an alternative computation of the classes, by means of a different (small) resolution, and also obtained partial positivity results. The resolution used in [AM09] has finitely many orbits of the Borel subgroup $B$, and this was a key fact in Huh's full proof of the positivity conjecture for CSM classes in that case Huh.

For generalized flag manifolds $G / B$ a resolution is given by Bott-Samelson varieties. These are iterated $\mathbb{P}^{1}$-bundles, and they can be constructed from any (possibly non-reduced) word consisting of simple reflections. Section 2.3 is devoted to the definition and cohomological properties of Bott-Samelson varieties. The key technical result in the paper is Theorem 3.3. establishing the necessary cohomological formulas calculating the pushforward of the Chern class of the logarithmic tangent bundle. The operators $\partial_{k}$ and $s_{k}$, and $\mathcal{T}_{k}=\partial_{k}-s_{k}$, appear naturally in these push-forward formulas. Properties of the operators $\mathcal{T}_{k}$ are discussed in $\$ 4$, and in $\$ 5$ we formulate the positivity conjecture and a related identity and provide partial evidence for these statements. In $\sqrt{6}$ we consider the torus-equivariant setting, and prove (Theorem 6.4) that a direct analogue of Theorem 1.1 holds for the equivariant CSM classes of Schubert cells. We also propose equivariant generalizations of the conjectures presented in $\$ 5$.

Equivariant Chern-Schwartz-MacPherson classes were defined by T. Ohmoto Ohm06] and have recently been the object of further study: A. Weber Web12 proved localization formulas for these classes, and in [RV] R. Rimányi and A. Varchenko use Weber's results to prove that equivariant CSM classes of Schubert cells agree with the $\kappa$ classes they studied in earlier work RTV14]. The $\kappa$ classes are related to the stable envelopes of D. Maulik and A. Okounkov [MO, which are classes in the equivariant cohomology of the cotangent bundle of flag manifolds. In future work we plan to investigate further the connection between the CSM classes and stable envelopes, both from the localization point of view (cf. [RV] and $\mathrm{Su}$ ) and from the point of view of Ginzburg's theory of bivariant Chern classes [Gin86].

Acknowledgements. We are grateful to Mark Shimozono for pointing out some algebraic identities between the operators $\partial_{k}$ and $s_{k}$; and to Dave Anderson, Jörg Schürmann, and Chanjiang Su for useful discussions.

\section{Preliminaries}

The goal of this section is to recall some basic facts on the cohomology of flag manifolds and on Bott-Samelson resolutions. We refer to [BK05], §2.1-§2.2, for more details and references to the standard literature.

2.1. Flag manifolds and Schubert varieties. Let $G$ be a complex simple Lie group and let $T \subseteq B \subseteq G$ be a maximal torus included in a Borel subgroup of $G$. Let $\mathfrak{h}$ and $\mathfrak{g}$ be the Lie algebras of $T$ and $G$, and let $R \subseteq \mathfrak{h}^{*}$ be the associated root system with the set of positive roots $R^{+}$determined by $B$. Denote by $\Delta:=\left\{\alpha_{1}, \ldots, \alpha_{r}\right\} \subseteq R^{+}$the set of simple roots. Let $R^{\vee}$ denote the set of coroots $\alpha^{\vee} \in \mathfrak{h}$ and $\langle\cdot, \cdot\rangle: R \otimes R^{\vee} \rightarrow \mathbb{Z}$ the evaluation pairing. 
To each root $\alpha \in R$ one associates a reflection $s_{\alpha}$ in the Weyl group $W=N_{G}(T) / T$. The set of simple reflections $s_{i}:=s_{\alpha_{i}}$ generate $W$, thus each $w \in W$ can be written as $w=s_{i_{1}} \cdots s_{i_{k}}$. The minimal such integer $k$ is denoted by $\ell(w)$, the length of $w$. If $k=\ell(w)$, then the decomposition $w=s_{i_{1}} \cdots s_{i_{k}}$ is said to be reduced. There is a partial order on $W$ called the Bruhat ordering, defined as follows: $u<v$ if there exists a chain

$$
u_{0}:=u \rightarrow u_{1}:=u s_{\beta_{1}} \rightarrow u_{2}:=u_{1} s_{\beta_{2}} \rightarrow \cdots \rightarrow u_{n}:=v=u_{n-1} s_{\beta_{n}}
$$

where the $\beta_{i}$ 's are roots in $R$ such that $\ell\left(u_{i}\right)>\ell\left(u_{i-1}\right)$. Let $G / B$ be the generalized flag variety. This is a homogeneous space for $G$ (hence a non-singular variety) and it is stratified by Schubert cells $X(w)^{\circ}:=B w B / B$, where $w \in W$, and each such cell $X(w)^{\circ}$ is isomorphic to $\mathbb{C}^{\ell(w)}$. The closure $X(w):=\overline{B w B / B}$ is called a Schubert variety. Each Schubert variety $X(w)$ has a fundamental class $[X(w)] \in H_{2 \ell(w)}(G / B)$, and these classes form a $\mathbb{Z}$-basis for the (co)homology. It may be verified that $X(v) \subseteq X(w)$ if and only if $v \leqslant w$ in the Bruhat order. In fact, $X(w)=\bigcup_{v \leqslant w} X(v)^{\circ}$. It follows (Ful98, Example 1.9.1]) that any class in $H_{*}(X(w))$ may be written uniquely as an integer linear combination $\sum_{v \leqslant w} c_{v}[X(v)]$.

Let $\mathfrak{h}_{\mathbb{Z}}^{*}$ be the integral weight lattice and let $\lambda \in \mathfrak{h}_{\mathbb{Z}}^{*}$ be an integral weight. Then one constructs the $G$-equivariant line bundle over $G / B$

$$
\mathcal{L}_{\lambda}:=G \times{ }^{B} \mathbb{C}_{-\lambda}=(G \times \mathbb{C}) / B
$$

where $B$ acts on $G \times \mathbb{C}$ by $b \cdot(g, u)=\left(g b^{-1}, \lambda(b)^{-1} u\right)$ (and the action of $B=U T$ on $\mathbb{C}$ extends the action of $T$ so that it is trivial over the unipotent group $U$ ).

The Chevalley formula states that

$$
c_{1}\left(\mathcal{L}_{\lambda}\right) \cdot[X(w)]=\sum\left\langle\lambda, \beta^{\vee}\right\rangle\left[X\left(w s_{\beta}\right)\right]
$$

where the sum is over all positive roots $\beta$ such that $\ell\left(w s_{\beta}\right)=\ell(w)-1$. See e.g., FW04, Lemma 8.1].

2.2. Two operators acting on $H^{*}(G / B)$. For each simple root $\alpha_{k} \in \Delta$ one can construct the BGG operator $\partial_{k}: H^{*}(G / B) \rightarrow H^{*}(G / B)$ defined in [BGG73] as follows. Let $P_{k} \subseteq G$ be the minimal parabolic subgroup corresponding to $\alpha_{k}$. Then the natural projection $\pi$ : $G / B \rightarrow G / P_{k}$ is a $\mathbb{P}^{1}$-bundle and there is a fiber square

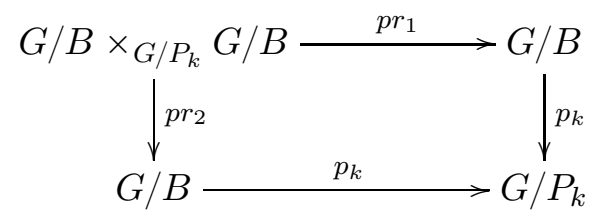

The BGG operator is defined to be $\partial_{k}=p_{k}^{*}\left(p_{k}\right)_{*}$. We record next two properties of this operator; see e.g., [Knu, Proposition 2] or [Tym08 for simple proofs.

Proposition 2.1. The operator $\partial_{k}$ satisfies the following properties:

(a) For all Weyl group elements $w \in W$,

$$
\partial_{k}([X(w)])= \begin{cases}0 & \text { if } \ell\left(w s_{k}\right)<\ell(w) \\ {\left[X\left(w s_{k}\right)\right]} & \text { if } \ell\left(w s_{k}\right)>\ell(w)\end{cases}
$$

In particular, $\partial_{k}^{2}=0$ and the $B G G$ operators satisfy the same braid relations as the elements of the Weyl group. 
(b) For all $\gamma_{1}, \gamma_{2} \in H^{*}(G / B)$,

$$
\partial_{k}\left(\gamma_{1} \gamma_{2}\right)=\partial_{k}\left(\gamma_{1}\right) \gamma_{2}+\gamma_{1} \partial_{k}\left(\gamma_{2}\right)-c_{1}\left(\mathcal{L}_{\alpha_{k}}\right) \partial_{k}\left(\gamma_{1}\right) \partial_{k}\left(\gamma_{2}\right)
$$

where $c_{1}\left(\mathcal{L}_{\alpha_{k}}\right)$ denotes the Chern class of $\mathcal{L}_{\alpha_{k}}$.

For each $w \in W$ there is a well defined map $r_{w}: G / T \rightarrow G / T$ obtained by multiplying on the right with any lift of $w$ in $N_{G}(T)$. This induces a ring endomorphism $r_{w}^{*}: H^{*}(G / T) \rightarrow$ $H^{*}(G / T)$. Note that the projection $G / T \rightarrow G / B$ is a $U \simeq B / T$-bundle and because $U$ is contractible this implies that the cohomology rings $H^{*}(G / B)$ and $H^{*}(G / T)$ are isomorphic. This defines a right action of $W$ on $H^{*}(G / B)$, denoted again by $w$; it will be clear from the context whether we refer to the Weyl group element or to its action on $H^{*}(G / B)$. It is well known (see e.g., [BGG73, §1]) that for $w=s_{k}$ this operator satisfies

$$
s_{k}=\mathrm{id}-c_{1}\left(\mathcal{L}_{\alpha_{k}}\right) \partial_{k}
$$

where the Chern class $c_{1}\left(\mathcal{L}_{\alpha_{k}}\right)$ acts on $H^{*}(G / B)$ by multiplication. Combining this with the Chevalley formula we obtain an identity

$$
s_{k}[X(w)]=\left\{\begin{array}{cl}
{[X(w)]} & \text { if } \ell\left(w s_{k}\right)<\ell(w) ; \\
-[X(w)]-\Sigma\left\langle\alpha_{k}, \beta^{\vee}\right\rangle\left[X\left(w s_{k} s_{\beta}\right)\right] & \text { if } \ell\left(w s_{k}\right)>\ell(w) .
\end{array}\right.
$$

where the sum is over all positive roots $\beta \neq \alpha_{k}$ such that $\ell(w)=\ell\left(w s_{k} s_{\beta}\right)$.

For use in $\varangle$ we also record the following commutation relation of the operators $\partial_{k}$ and $s_{k}$.

Lemma 2.2. With notation as above,

$$
s_{k} \partial_{k}=\partial_{k} \quad, \quad \partial_{k} s_{k}=-\partial_{k}
$$

In particular, $\partial_{k} s_{k}+s_{k} \partial_{k}=0$.

Proof. The first equality follows immediately from the definition of $s_{k}$ and the fact that $\partial_{k}^{2}=0$. The second equality is a consequence of Proposition 2.1(b):

$$
\begin{aligned}
\partial_{k} s_{k} & =\partial_{k}-\partial_{k}\left(c_{1}\left(\mathcal{L}_{\alpha_{k}}\right) \partial_{k}\right) \\
& =\partial_{k}-\partial_{k}\left(c_{1}\left(\mathcal{L}_{\alpha_{k}}\right)\right) \partial_{k}-c_{1}\left(\mathcal{L}_{\alpha_{k}}\right) \partial_{k}^{2}+c_{1}\left(\mathcal{L}_{\alpha_{k}}\right) \partial_{k}\left(c_{1}\left(\mathcal{L}_{\alpha_{k}}\right)\right) \partial_{k}^{2} \\
& =\partial_{k}-\partial_{k}\left(c_{1}\left(\mathcal{L}_{\alpha_{k}}\right)\right) \partial_{k} \\
& =-\partial_{k},
\end{aligned}
$$

where we used the fact that $\partial_{k}\left(c_{1}\left(\mathcal{L}_{\alpha_{k}}\right)\right)=\left\langle\alpha_{k}, \alpha_{k}^{\vee}\right\rangle=2$ as may be checked using Proposition 2.1(a) and the Chevalley formula.

2.3. Bott-Samelson varieties. For each word $s_{i_{1}} s_{i_{2}} \ldots s_{i_{k}}$ for an element $w \in W$ one can construct a tower of $\mathbb{P}^{1}$ bundles, the Bott-Samelson variety $Z:=Z_{i_{1}, \ldots, i_{k}}$, endowed with a map $\theta:=\theta_{i_{1}, \ldots, i_{k}}: Z \rightarrow X(w)$. If the word is reduced, this map is birational, giving a resolution of singularities for $X(w)$ (depending on the choice of the word for $w$ ). There are several ways to do this, but for our purpose we present an inductive construction which can be found e.g., in [BK05, §2.2].

If the word is empty, then define $Z:=p t$. In general assume we have constructed $Z^{\prime}:=Z_{i_{1}, \ldots, i_{k-1}}$ and the map $\theta^{\prime}: Z^{\prime} \rightarrow X\left(w^{\prime}\right)$, for $w^{\prime}=s_{i_{1}} \cdots s_{i_{k-1}}$. Define $Z=Z_{i_{1}, \ldots, i_{k}}$ so that the left square in the diagram

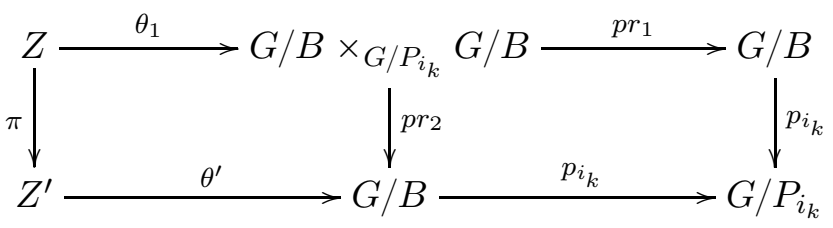


is a fiber square; the morphisms $p r_{1}, p r_{2}, p_{i_{k}}$ are the natural projections. From this construction it follows that $Z_{i_{1}, \ldots, i_{k}}$ is a smooth, projective variety of dimension $k$.

The Bott-Samelson variety $Z$ is equipped with a simple normal crossing (SNC) divisor $D_{Z}$. We recall next an explicit inductive construction of this divisor, which will be needed later. If $Z=p t$, then $D_{Z}=\varnothing$. In general, $G / B$ is the projectivization $\mathbb{P}(E)$ of a homogeneous rank-2 vector bundle $E \rightarrow G / P_{k}$, defined up to tensoring with a line bundle. Define $\mathcal{E}:=E \otimes \mathcal{O}_{E}(1)$, a vector bundle over $G / B=\mathbb{P}(E)$. Then we have the Euler sequence of the projective bundle $\mathbb{P}(E)$

$$
0 \longrightarrow \mathcal{O}_{\mathbb{P}(E)} \longrightarrow \mathcal{E} \longrightarrow \mathcal{Q} \longrightarrow 0
$$

where $\mathcal{Q}$ is the relative tangent bundle $T_{p_{i_{k}}}$. Note that $\mathcal{E}$ is independent of the specific choice of $E$, and $p r_{2}: G / B \times_{G / P_{i_{k}}} G / B \rightarrow G / B$, that is, the pull-back of $\mathbb{P}(E)$ via $p_{i_{k}}$, may be identified with $\mathbb{P}(\mathcal{E})$. Let $\mathcal{E}^{\prime}:=\left(\theta^{\prime}\right)^{*} \mathcal{E}$ and $\mathcal{Q}^{\prime}:=\left(\theta^{\prime}\right)^{*} \mathcal{Q}$, and pull-back the previous sequence via $\theta^{\prime}$ to get an exact sequence

$$
0 \longrightarrow \mathcal{O}_{Z^{\prime}} \longrightarrow \mathcal{E}^{\prime} \longrightarrow \mathcal{Q}^{\prime} \longrightarrow 0 \text {. }
$$

The inclusion $\mathcal{O}_{Z^{\prime}} \hookrightarrow \mathcal{E}^{\prime}$ gives a section $\sigma: Z^{\prime} \rightarrow Z$ of $\pi$ and therefore a divisor $D_{k}:=$ $\sigma\left(Z^{\prime}\right)=\mathbb{P}\left(\mathcal{O}_{Z^{\prime}}\right)$ in $\mathbb{P}\left(\mathcal{E}^{\prime}\right)=Z$. The SNC divisor on $Z$ is defined by

$$
D_{Z}=\pi^{-1}\left(D_{Z^{\prime}}\right) \cup D_{k}
$$

where $D_{Z^{\prime}}$ is the inductively constructed SNC divisor on $Z^{\prime}$. The following result is well known, see e.g., [BK05, §2.2]:

Proposition 2.3. If $w$ is a reduced word, then the image of the composition $\theta=p r_{1} \circ \theta_{1}$ : $Z_{i_{1}, \ldots, i_{k}} \rightarrow G / B$ is the Schubert variety $X(w)$. Moreover, $\theta^{-1}\left(X(w) \backslash X(w)^{\circ}\right)=D_{Z_{i_{1}, \ldots, i_{k}}}$ and the restriction map

$$
\theta: Z_{i_{1}, \ldots, i_{k}} \backslash D_{Z_{i_{1}, \ldots, i_{k}}} \rightarrow X(w)^{\circ}
$$

is an isomorphism.

Let $h_{k}:=c_{1}\left(\mathcal{O}_{\mathcal{E}^{\prime}}(1)\right) \in H^{2}(Z)$. For later use we record next the class of the divisor $D_{k}$ in $Z$, and the Chern classes of the relative tangent bundle $T_{\pi}=T_{\mathbb{P}\left(\mathcal{E}^{\prime}\right) \mid Z^{\prime}}$ and of $T_{Z}$.

Proposition 2.4. The following identities hold in $H^{*}(Z)$ :

(a) $D_{k}=c_{1}\left(\pi^{*}\left(\mathcal{Q}^{\prime}\right) \otimes \mathcal{O}_{\mathcal{E}^{\prime}}(1)\right) \in H^{*}(Z)$;

(b) $h_{k} \cdot D_{k}=0$;

(c) $c\left(T_{\pi}\right)=\left(1+D_{k}\right)\left(1+h_{k}\right)$, and therefore

$$
c\left(T_{Z}\right)=\pi^{*}\left(c\left(T_{Z^{\prime}}\right)\right)\left(1+h_{k}\right)\left(1+D_{k}\right) .
$$

Proof. (a) follows from the definition of $D_{k}$ and [Ful98, Ex. 3.2.17], since $\mathcal{Q}^{\prime}=\mathcal{E}^{\prime} / \mathcal{O}_{Z^{\prime}}$.

(b) holds since $\left.h_{k}\right|_{D_{k}}=c_{1}\left(\mathcal{O}_{\mathcal{O}_{Z^{\prime}}}(1)\right)=0$.

To prove (c), note that by (7) the Chern roots of $\mathcal{E}^{\prime}$ are 0 and $c_{1}\left(\mathcal{Q}^{\prime}\right)$; it follows from (a) that the Chern roots of $\pi^{*} \mathcal{E}^{\prime} \otimes \mathcal{O}_{\mathcal{E}^{\prime}}(1)$ are $h_{k}$ and $D_{k}$. The Euler sequence

$$
0 \longrightarrow \mathcal{O}_{Z} \longrightarrow \pi^{*} \mathcal{E}^{\prime} \otimes \mathcal{O}_{\mathcal{E}^{\prime}}(1) \longrightarrow T_{\pi} \longrightarrow 0
$$

then implies $c\left(T_{\pi}\right)=c\left(\pi^{*} \mathcal{E}^{\prime} \otimes \mathcal{O}_{\mathcal{E}^{\prime}}(1)\right)=\left(1+h_{k}\right)\left(1+D_{k}\right)$. The last statement follows from $c\left(T_{Z}\right)=\pi^{*}\left(c\left(T_{Z^{\prime}}\right)\right) c\left(T_{\pi}\right)$. 


\section{Chern-Schwartz-MacPherson classes of Schubert cells in $G / B$}

3.1. CSM classes. Let $Y$ be an algebraic variety over $\mathbb{C}$. Denote by $\mathcal{F}(Y)$ the group of constructible functions on $Y$ : the elements of $\mathcal{F}(Y)$ are finite sums $\sum c_{i} \mathbb{1}_{W_{i}}$ where $c_{i} \in \mathbb{Z}$, $W_{i} \subseteq Y$ are locally closed subvarieties, and $\mathbb{1}_{W}$ denotes the characteristic function taking value 1 on $p \in W$ and 0 otherwise. If $f: Y \rightarrow X$ is a proper morphism of varieties, one can define a push-forward $f_{*}: \mathcal{F}(Y) \rightarrow \mathcal{F}(X)$ by setting

$$
f_{*}\left(\mathbb{1}_{W}\right)(p)=\chi\left(f^{-1}(p) \cap W\right)
$$

for $W \subseteq Y$ a subvariety and $p \in X$, and extending by linearity to every $\varphi \in \mathcal{F}(Y)$; this makes $\mathcal{F}$ into a covariant functor. Here $\chi$ denotes the topological Euler characteristic. MacPherson Mac74 proved a conjecture of Deligne and Grothendieck stating that there exists a natural transformation $c_{*}: \mathcal{F} \rightarrow H_{*}$ such that if $Y$ is non-singular, then $c_{*}\left(\mathbb{1}_{Y}\right)=c\left(T_{Y}\right) \cap[Y]$. The naturality of $c_{*}$ means that if $f: Y \rightarrow X$ is a proper morphism, then the following diagram commutes:

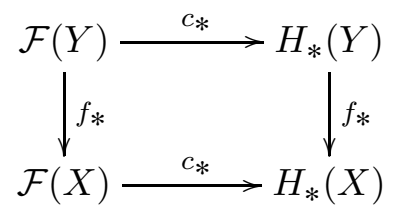

That is,

$$
f_{*}\left(c_{*}(\varphi)\right)=c_{*}\left(f_{*}(\varphi)\right)
$$

in $H_{*}(X)$, for all constructible functions $\varphi$. Resolution of singularities and the normalization requirement easily imply that $c_{*}$ is unique.

If $Y$ is a compact complex variety, the class $c_{*}\left(\mathbb{1}_{Y}\right)$ coincides with a class defined earlier by M. H. Schwartz [Sch65a, Sch65b]; this class is the Chern-Schwartz-MacPherson (CSM) class of $Y$. Taking $f$ to be a constant map, the commutativity of the above diagram implies that $\int c_{*}\left(\mathbb{1}_{Y}\right)=\chi(Y)$, so this class provides a natural generalization of the Poincaré-Hopf theorem to possibly singular varieties. Abusing language a little, we denote by $c_{\mathrm{SM}}(W):=$ $c_{*}\left(1_{W}\right) \in H_{*} Y$ the CSM class of any constructible set $W$ in a variety $Y$; by additivity of Euler characteristics, $\int c_{\mathrm{SM}}(W)=\chi(W)$.

Our main tool will be the observation that if $Z$ is a nonsingular variety and $W \subseteq Z$ is an open subvariety such that $Z \backslash W$ is a SNC divisor with components $D_{i}$, then

$$
c_{\mathrm{SM}}(W)=\frac{c\left(T_{Z}\right)}{\prod_{i}\left(1+D_{i}\right)} \cap[Z] \in H_{*} Z
$$

(cf. [GP02, Proposition 15.3], [Alu99, Theorem 1]).

In fact this observation may be used to extend the scope of the natural transformation $c_{*}$ to arbitrary algebraically closed fields of characteristic 0 , with values in the Chow group $A_{*}$. In this generality, $c_{*}$ may be constructed as follows. Every constructible function on $Y$ can be written as a linear combination of characteristic functions $\mathbb{1}_{W}$ for $W$ locally closed and non-singular in $Y$, so it suffices to describe $c_{\mathrm{SM}}(W)=c_{*}\left(\mathbb{1}_{W}\right)$ for such $W$. By resolution of singularities, there exists a desingularization $\pi: Z \rightarrow \bar{W}$ of the closure $\bar{W}$ of $W$ in $Y$ such that $D:=\pi^{-1}(\bar{W} \backslash W)$ is a SNC divisor in $Z$. Then one may take the push-forward of (9) to $Y$ as the definition of $c_{*}\left(\mathbb{1}_{W}\right)$ : one can show that over algebraically closed fields of characteristic 0 the resulting $c_{*}$ is independent of the choices and satisfies the DeligneGrothendieck axioms mentioned above ([Alu06a, Alu06b]). 
3.2. A recursive formula for CSM classes of Schubert cells. We will now apply identity (9) to calculate the Chern-Schwartz-MacPherson class of a Schubert cell $X(w)^{\circ} \subseteq$ $G / B$. This class may be viewed as an element of $H_{*}(G / B)$, and in fact of $H_{*}(X(w))$, and hence it can be written as an integer linear combination of classes $[X(v)]$ for $v \leqslant w$ in the Bruhat order, as we observed in \$2.1. We will give an algorithm which yields this linear combination. All the necessary ingredients were developed in $\$ 2.3$ and we keep the notation of that section. In particular we recall the fiber diagram (5):

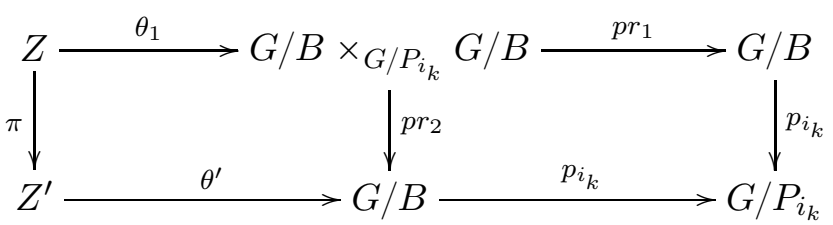

Let $s_{i_{1}} \cdots s_{i_{k}}$ be any word (reduced or otherwise), and let $Z:=Z_{i_{1}, \ldots, i_{k}}$ be the corresponding Bott-Samelson variety. Recall from 92.3 that $G / B \times{ }_{G / P_{i_{k}}} G / B=\mathbb{P}(\mathcal{E})$ for a canonically defined vector rank-2 vector bundle $\mathcal{E}$ on $G / B$; thus $Z=\mathbb{P}\left(\mathcal{E}^{\prime}\right)$ is the projectivization of the pull-back $\mathcal{E}^{\prime}=\theta^{\prime *}(\mathcal{E})$. In $H^{2}\left(Z_{i_{1}, \ldots, i_{k}}\right)$ we have the tautological class $h_{k}=c_{1}\left(\mathcal{O}_{\mathcal{E}^{\prime}}(1)\right)$, as well as the pull-backs of $h_{j}$ from $Z_{i_{1}, \ldots, i_{j}}$ for $j<k$; we will omit the pull-back notation.

Let $D_{Z}$ be the SNC divisor defined in 92.3 , and denote by $Z^{\circ}=Z_{i_{1}, \ldots, i_{k}}^{\circ}$ the complement $Z \backslash D_{Z}$. By (9),

$$
c_{\mathrm{SM}}\left(Z^{\circ}\right)=\frac{c\left(T_{Z}\right)}{\left(1+D_{1}\right) \cdots\left(1+D_{k}\right)} \cap[Z]
$$

where $D_{1}, \ldots, D_{k}$ are the components of $D_{Z}$.

Lemma 3.1. With notation as above, the following holds in $H_{*}(Z)$ :

$$
c_{S M}\left(Z^{\circ}\right)=\left(1+h_{k}\right) \cdot \pi^{*}\left(c_{S M}\left(Z^{\prime \circ}\right)\right)=\prod_{j=1}^{k}\left(1+h_{j}\right) \cdot[Z] .
$$

Proof. The first formula follows from (10) and Proposition 2.4(c), and the second formula is an immediate consequence.

Now let $X(w)$ be the Schubert variety determined by $w \in W$, that is, the closure of $X(w)^{\circ}$ in $G / B$, and fix a reduced decomposition $s_{i_{1}} \cdots s_{i_{k}}$ of $w$ and the corresponding Bott-Samelson variety $Z$. As recalled in Proposition 2.3, the composition $p r_{1} \circ \theta_{1}$ gives a proper birational morphism (hence a desingularization) $\theta: Z \rightarrow X(w)$, restricting to an isomorphism on $\theta^{-1}\left(X(w)^{\circ}\right)=Z^{\circ}$.

Lemma 3.2. The CSM class of the Schubert cell $X(w)^{\circ}$ is given by

$$
c_{S M}\left(X(w)^{\circ}\right)=\theta_{*}\left(\left(1+h_{k}\right) \cdot \pi^{*}\left(c_{S M}\left(Z^{\prime \circ}\right)\right)\right) .
$$

Proof. By construction, $\theta_{*}\left(\mathbb{1}_{Z^{\circ}}\right)=\mathbb{1}_{X(w)^{\circ}}$; therefore, the functoriality of CSM classes (8) implies $c_{\mathrm{SM}}\left(X(w)^{\circ}\right)=\theta_{*}\left(c_{\mathrm{SM}}\left(Z^{\circ}\right)\right)$, and the stated formula then follows from Lemma 3.1 .

Lemma 3.2 motivates the study of the quantity $\theta_{*}\left(\left(1+h_{k}\right) \cdot \pi^{*}(\gamma)\right)$ for $\gamma \in H_{*}\left(Z^{\prime}\right)$. The next theorem gives the key formulas needed for explicit calculations, in terms of the operators introduced in 92.2 .

Theorem 3.3. Let $\gamma \in H_{*}\left(Z^{\prime}\right)$. Then the following holds in $H_{*}(G / B)$ :

(a) $\theta_{*}\left(\pi^{*}(\gamma)\right)=\partial_{i_{k}}\left(\theta_{*}^{\prime}(\gamma)\right)$; 
(b) $\theta_{*}\left(h_{k} \cdot \pi^{*}(\gamma)\right)=-s_{i_{k}}\left(\theta_{*}^{\prime}(\gamma)\right)$.

Therefore,

$$
\theta_{*}\left(\left(1+h_{k}\right) \cdot \pi^{*}(\gamma)\right)=\mathcal{T}_{i_{k}}\left(\theta_{*}^{\prime}(\gamma)\right)
$$

where $\mathcal{T}_{i}: H^{*}(G / B) \rightarrow H^{*}(G / B)$ is the operator given by $\mathcal{T}_{i}=\partial_{i}-s_{i}$.

Before proving the theorem, we note that if $\gamma=c_{\mathrm{SM}}\left(Z^{\prime \circ}\right)$, then $\theta_{*}^{\prime}(\gamma)=c_{\mathrm{SM}}\left(X\left(w^{\prime}\right)^{\circ}\right)$ where $w^{\prime}=s_{i_{1}} \cdots s_{i_{k-1}}=w s_{i_{k}}$. Therefore Theorem 3.3 gives a recursive formula to calculate the CSM classes:

Corollary 3.4. Let $w \in W$ be a non-identity element and let $s_{k}$ be a simple reflection such that $\ell\left(w s_{k}\right)<\ell(w)$. Then the following recursive identity holds:

$$
c_{S M}\left(X(w)^{\circ}\right)=\mathcal{T}_{k}\left(c_{S M}\left(X\left(w s_{k}\right)^{\circ}\right)\right),
$$

with the initial condition that $c_{S M}\left(X(i d)^{\circ}\right)=c_{S M}(p t)=[p t]$.

The explicit action of the operator $\mathcal{T}_{k}$ on Schubert classes $[X(u)]$ is obtained by combining identities (2) and (44) above. The resulting formula together with other properties of the operator $\mathcal{T}_{k}$ will be presented in $\$ 4$ below.

Proof of Theorem 3.3. Both the left and right squares in (5) are fiber squares, and $p_{i_{k}}$ is flat and $\theta=p r_{1} \theta_{1}$ is proper, so

$$
\theta_{*} \pi^{*}(\gamma)=p_{i_{k}}^{*}\left(p_{i_{k}}\right)_{*} \theta_{*}^{\prime}(\gamma)=\partial_{i_{k}}\left(\theta_{*}^{\prime}(\gamma)\right)
$$

by [Ful98, Proposition 1.7] and the definition of $\partial_{i_{k}}$ given in 92.2 . This proves (a).

For $(\mathrm{b})$, let $\underline{\gamma}:=\theta_{*}^{\prime}(\gamma) \in H^{*}(G / B)$ and

$$
\tilde{h}_{k}=c_{1}\left(\mathcal{O}_{\mathcal{E}}(1)\right) \in H^{2}\left(G / B \times_{G / P_{i_{k}}} G / B\right),
$$

so that $h_{k}=\theta_{1}^{*}\left(\tilde{h}_{k}\right)$. Then

$$
\begin{aligned}
\theta_{*}\left(h_{k} \cdot \pi^{*}(\gamma)\right) & =\left(p r_{1}\right)_{*}\left(\theta_{1}\right)_{*}\left(\theta_{1}^{*}\left(\tilde{h}_{k}\right) \cdot \pi^{*}(\gamma)\right) \\
& =\left(p r_{1}\right)_{*}\left(\tilde{h}_{k} \cdot\left(\theta_{1}\right)_{*} \pi^{*}(\gamma)\right) \\
& =\left(p r_{1}\right)_{*}\left(\tilde{h}_{k} \cdot p r_{2}^{*}\left(\theta^{\prime}\right)_{*}(\gamma)\right) \\
& =\left(p r_{1}\right)_{*}\left(\tilde{h}_{k} \cdot p r_{2}^{*}(\underline{\gamma})\right) .
\end{aligned}
$$

In the second equality we used the projection formula, and in the third we used the fact that the left square in (5) is a fiber square and that $p r_{2}$ is flat and $\theta^{\prime}$ is proper. Now recall that $G / B$ is the projectivization $\mathbb{P}(E)$ of a vector bundle $E$ over $G / P_{i_{k}}$, and $\mathcal{E}$ is $p_{i_{k}}^{*}(E) \otimes \mathcal{O}_{E}(1)$ as a bundle over $G / B$. We can compute the tautological subbundle $\mathcal{O}_{\mathcal{E}}(-1)$ of $p r_{2}^{*}(\mathcal{E})$, a bundle over $G / B \times{ }_{G / P_{i_{k}}} G / B$, by using [Ful98, Appendix B.5.5]:

$$
\mathcal{O}_{\mathcal{E}}(-1)=\mathcal{O}_{p_{i_{k}}^{*}(E) \otimes \mathcal{O}_{E}(1)}(-1)=p r_{2}^{*} \mathcal{O}_{p_{i_{k}}^{*}(E)}(-1) \otimes p r_{2}^{*} \mathcal{O}_{E}(1)=p r_{1}^{*} \mathcal{O}_{E}(-1) \otimes p r_{2}^{*} \mathcal{O}_{E}(1)
$$

Letting $\eta=c_{1}\left(\mathcal{O}_{E}(1)\right)$, this implies

$$
\tilde{h}_{k}=c_{1}\left(p r_{1}^{*} \mathcal{O}_{E}(1)\right)+c_{1}\left(p r_{2}^{*} \mathcal{O}_{E}(-1)\right)=p r_{1}^{*}(\eta)-p r_{2}^{*}(\eta),
$$

and the projection formula gives

$$
\begin{aligned}
\left(p r_{1}\right)_{*}\left(\tilde{h}_{k} \cdot p r_{2}^{*}(\underline{\gamma})\right) & =\left(p r_{1}\right)_{*}\left(\left(p r_{1}^{*}(\eta)-p r_{2}^{*}(\eta)\right) \cdot p r_{2}^{*}(\underline{\gamma})\right) \\
& =\eta \cdot\left(p r_{1}\right)_{*} p r_{2}^{*}(\underline{\gamma})-\left(p r_{1}\right)_{*} p r_{2}^{*}(\eta \cdot \underline{\gamma}) \\
& =\eta \cdot p_{i_{k}}^{*}\left(p_{i_{k}}\right)_{*}(\underline{\gamma})-p_{i_{k}}^{*}\left(p_{i_{k}}\right)_{*}(\eta \cdot \underline{\gamma})
\end{aligned}
$$


where the last equality follows since the second square in (5) is also a fiber square and $p_{i_{k}}$ is both flat and proper. By definition, $\partial_{i_{k}}=p_{i_{k}}^{*}\left(p_{i_{k}}\right)_{*}$. Putting all together, we have shown that

$$
\theta_{*}\left(h_{k} \cdot \pi^{*} \gamma\right)=\eta \cdot \partial_{i_{k}}(\underline{\gamma})-\partial_{i_{k}}(\eta \cdot \underline{\gamma})
$$

Since $\mathcal{O}_{E}(1)$ has degree 1 on the fibers of $p_{i_{k}}$, and $p_{i_{k}}$ has relative dimension 1 , we have

$$
\partial_{i_{k}}(\eta)=p_{i_{k}}^{*}\left(p_{i_{k}}\right)_{*}(\eta)=[G / B] \text {. }
$$

We use this and part (b) of Proposition 2.1 to get

$$
\begin{aligned}
\partial_{i_{k}}(\eta \cdot \underline{\gamma}) & =\partial_{i_{k}}(\eta) \cdot \underline{\gamma}+\eta \cdot \partial_{i_{k}}(\underline{\gamma})-c_{1}\left(\mathcal{L}_{\alpha_{i_{k}}}\right) \cdot \partial_{i_{k}}(\eta) \cdot \partial_{i_{k}}(\underline{\gamma}) \\
& =\underline{\gamma}+\eta \cdot \partial_{i_{k}}(\underline{\gamma})-c_{1}\left(\mathcal{L}_{\alpha_{i_{k}}}\right) \cdot \partial_{i_{k}}(\underline{\gamma})
\end{aligned}
$$

and finally

$$
\theta_{*}\left(h_{k} \cdot \pi^{*} \gamma\right)=-\underline{\gamma}+c_{1}\left(\mathcal{L}_{\alpha_{i_{k}}}\right) \cdot \partial_{i_{k}}(\underline{\gamma})=\left(-\mathrm{id}+c_{1}\left(\mathcal{L}_{\alpha_{i_{k}}}\right)\right)(\underline{\gamma})=-s_{i_{k}}(\underline{\gamma})
$$

by (3), concluding the proof of (b).

3.3. Chern classes of Schubert cells in $G / P$. Fix a parabolic subgroup $P \subset G$ containing the Borel subgroup $B$. Let $W_{P} \subseteq W$ be the subgroup generated by the simple reflections in $P$. It is known (see e.g., Hum90, $\S 1.10]$ ) that each coset in $W / W_{P}$ has a unique minimal length representative; we denote by $W^{P}$ the set of these representatives. If $w \in W$, then one can define a length function $\ell: W / W_{P} \rightarrow \mathbb{N}$ by $\ell\left(w W_{P}\right):=\ell\left(w^{\prime}\right)$ where $w^{\prime} \in W^{P}$ is in the coset of $w$.

The space $G / P$ is a projective manifold of dimension $\ell\left(w_{0} W_{P}\right)$, where $w_{0}$ is the longest element in $W$. For each $w \in W^{P}$ there is a Schubert cell $X\left(w W_{P}\right)^{\circ}:=B w P / P$ of dimension $\ell\left(w W_{P}\right)$, and the corresponding Schubert variety $X\left(w W_{P}\right):=\overline{B w P / P}$; see e.g., [BL00, $\S 2.6]$. The fundamental classes $\left[X\left(w W_{P}\right)\right] \in H_{2 \ell\left(w W_{P}\right)}(G / P)\left(w \in W^{P}\right)$ form a $\mathbb{Z}$-basis for the homology $H_{*}(G / P)$. The natural projection $p: G / B \rightarrow G / P$ satisfies $p(X(w))=$ $X\left(w W_{P}\right)$ and the induced map in homology is given by

$$
p_{*}[X(w)]= \begin{cases}{\left[X\left(w W_{P}\right)\right]} & \text { if } \ell(w)=\ell\left(w W_{P}\right) ; \\ 0 & \text { otherwise } .\end{cases}
$$

Proposition 3.5. With notation as above,

$$
c_{S M}\left(X\left(w W_{P}\right)^{\circ}\right)=p_{*}\left(c_{S M}\left(X(w)^{\circ}\right)\right) \in H_{*}(G / P)
$$

for all $w \in W$. Further, if $u \leqslant w$ and $\ell(u)=\ell\left(u W_{P}\right)$, then the coefficient of $[X(u)]$ in $c_{S M}\left(X(w)^{\circ}\right)$ equals the coefficient of $\left[X\left(u W_{P}\right)\right]$ in $c_{S M}\left(X\left(w W_{P}\right)^{\circ}\right)$.

Proof. The topological Euler characteristic $\chi$ of the fibers of the restriction of $p$ to $X(w)^{\circ}$ is constant, hence the push-forward $p_{*}\left(\mathbb{1}_{X(w)^{\circ}}\right)$ equals $\chi \cdot \mathbb{1}_{X\left(w W_{P}\right)^{\circ}}$. By functoriality of CSM classes (8) this implies that $p_{*}\left(c_{\mathrm{SM}}\left(X(w)^{\circ}\right)\right)=\chi \cdot c_{\mathrm{SM}}\left(X\left(w W_{P}\right)^{\circ}\right)$. Since the coefficient of $[p t]$ in both CSM classes equals 1 , it follows that $\chi=1$. The last claim follows from (11).

Thus the CSM classes of Schubert cells in $G / P$ are determined by the corresponding classes in $G / B$. For example, the CSM classes of Schubert cells in the ordinary Grassmannian, determined explicitly in [AM09], can also be computed in principle using the recursive formula obtained in Corollary 3.4, see Example 4.4 for a concrete example. Further, the push-forward formula (11) implies that if the positivity conjecture discussed in $\$ 5$ is true for the CSM classes of Schubert cells in $G / B$, then the analogous conjecture must be true for CSM classes of Schubert cells in $G / P$ for any parabolic $P$ containing $B$. 


\section{The operators $\mathcal{T}_{k}$ and a Weyl group representation on $H^{*}(G / B)$}

In this section we analyze the operator $\mathcal{T}_{k}=\partial_{k}-s_{k}: H_{*}(G / B) \mapsto H_{*}(G / B)$ which gives the recursion for CSM classes of Schubert cells as proven in Corollary 3.4. We start by recording the main algebraic properties of the operators $\mathcal{T}_{k}$.

Proposition 4.1. The following identities hold:

(a) $\mathcal{T}_{k}^{2}=1$

(b) The operators $\mathcal{T}_{k}$ satisfy the braid relations, i.e., $\left(\mathcal{T}_{i} \mathcal{T}_{j}\right)^{m_{i, j}}=1$ where $m_{i, j}$ is the order of the element $s_{i} s_{j} \in W$. Also, if $w=s_{i_{1}} \cdots s_{i_{k}}$ is a representation of an element $w \in W$ as a word in simple reflections, then the operator $\mathcal{T}_{w}:=\mathcal{T}_{i_{1}} \cdots \mathcal{T}_{i_{k}}$ is independent of the choice of the word representing $w$.

(c) For any $u, v \in W, \mathcal{T}_{u} \cdot \mathcal{T}_{v}=\mathcal{T}_{u v}$.

Proof. First we note that

$$
\mathcal{T}_{k}^{2}=\left(\partial_{k}-s_{k}\right)^{2}=s_{k}^{2}=1
$$

since $\partial_{k}^{2}=0$ and $\partial_{k} s_{k}+s_{k} \partial_{k}=0$ by Lemma 2.2. This proves part (a). To prove the first part of (b), it suffices to show that the relations hold after applying the operators to the classes $c_{\mathrm{SM}}\left(X(w)^{\circ}\right)$, since these form a basis for $H_{*}(G / B)$. These relations follow then immediately from the fact that for all $w \in W$ and all simple reflections $s_{k}$,

$$
\mathcal{T}_{k}\left(c_{\mathrm{SM}}\left(X(w)^{\circ}\right)\right)=c_{\mathrm{SM}}\left(X\left(w s_{k}\right)^{\circ}\right)
$$

as a consequence of Corollary 3.4 and part (a). The independence of $\mathcal{T}_{w}$ on the specific word for $w$ is also an immediate consequence of (13). Finally, (c) follows from the independence of $\mathcal{T}_{w}$ on the word representing $w$.

The proposition implies that the operators $\mathcal{T}_{w}$ define a representation of the Weyl group $W$ on $H_{*}(G / B)$. We record an immediate consequence of the identity (13) from the proof of Proposition 4.1 .

Corollary 4.2. Let $u, w$ be two Weyl group elements. Then the identity

$$
\mathcal{T}_{u}\left(c_{S M}\left(X(w)^{\circ}\right)\right)=c_{S M}\left(X\left(w u^{-1}\right)^{\circ}\right)
$$

holds in $H_{*}(G / B)$. In particular, $c_{S M}\left(X(w)^{\circ}\right)=\mathcal{T}_{w^{-1}}([p t])$.

Combining the actions of $\partial_{k}$ and $s_{k}$ on Schubert classes found in the identities (2) and (4) from $\$ 2.2$ we obtain the following explicit formula for $\mathcal{T}_{k}$ :

\section{Proposition 4.3.}

$$
\mathcal{T}_{k}([X(w)])=\left\{\begin{array}{cc}
-[X(w)] & \text { if } \ell\left(w s_{k}\right)<\ell(w) \\
{\left[X\left(w s_{k}\right)\right]+[X(w)]+\Sigma\left\langle\alpha_{k}, \beta^{\vee}\right\rangle\left[X\left(w s_{k} s_{\beta}\right)\right]} & \text { if } \ell\left(w s_{k}\right)>\ell(w)
\end{array}\right.
$$

where the sum is over all positive roots $\beta \neq \alpha_{k}$ such that $\ell(w)=\ell\left(w s_{k} s_{\beta}\right)$.

Example 4.4. Using Corollary 4.2 and Proposition 4.3 it is straightforward to implement computations of CSM classes of Schubert cells in symbolic manipulation packages such as Maple. For instance, we obtain that the CSM class for the open cell in the flag manifold $\mathrm{Fl}(4)$ (in type A) is:

$$
\begin{gathered}
c_{\mathrm{SM}}\left(X(4321)^{\circ}\right)=[X(4321)]+[X(4312)]+[X(4231)]+[X(3421)]+2[X(4213)]+2[X(4132)] \\
+[X(3412)]+2[X(3241)]+2[X(2431)]+[X(4123)]+5[X(3214)]+5[X(3142)] \\
+3[X(2413)]+[X(2341)]+5[X(1432)]+3[X(3124)]+4[X(2314)]+6[X(2143)] \\
\quad+4[X(1423)]+3[X(1342)]+3[X(2134)]+4[X(1324)]+3[X(1243)]+[X(1234)]
\end{gathered}
$$


where we use the standard identification of the elements of $W$ with permutations in indexing the 4 ! Schubert classes.

Note that the terms corresponding to the 'Grassmannian permutations' $\left(a_{1} a_{2} b_{1} b_{2}\right)$ with $a_{1}<a_{2}$ and $b_{1}<b_{2}$ are

$$
[X(3412)]+3[X(2413)]+4[X(1423)]+4[X(2314)]+4[X(1324)]+[X(1234)]
$$

and push-forward as prescribed by identity (12) in 93.3 to the CSM class for the open cell in $G(2,4)$ (cf. the row corresponding to $\square$ in [AM09, Example 1.2]).

Remark 4.5. Even if $\ell\left(w s_{k}\right)>\ell(w), \mathcal{T}_{k}([X(w)])$ is in general not a positive combination of Schubert classes. For example, let $G=\mathrm{SL}_{4}(\mathbb{C})$, and let $w=w_{0} s_{3}$, where $w_{0}$ is the longest element in $W=S_{4}$, the symmetric group with 4 letters. Using again the standard identification of the elements of $W$ with permutations, so that $w_{0}=(4321)$ and $s_{3}=(1243)$, then $w=(4312)$ and

$$
\mathcal{T}_{3}([X(4312)])=[X(4312)]+[X(4321)]-[X(4231)] .
$$

Nevertheless, substantial evidence suggests that the classes $\mathcal{T}_{k}(\gamma)$, and hence all classes $\mathcal{T}_{w}(\gamma)$, are positive linear combinations of Schubert classes if $\gamma$ is a positive combination of CSM classes $c_{\mathrm{SM}}\left(X(u)^{\circ}\right)$; see $\$ 5$.

\section{Positivity of CSM Classes}

Fix $w \in W$ and consider the CSM class $c_{\mathrm{SM}}\left(X(w)^{\circ}\right)$. As we have shown, if $s_{i_{1}} \cdots s_{i_{k}}$ is a reduced decomposition for $w$, then

$$
c_{\mathrm{SM}}\left(X(w)^{\circ}\right)=\theta_{*}\left(c_{\mathrm{SM}}\left(Z^{\circ}\right)\right)=\theta_{*}\left(\prod_{j=1}^{k}\left(1+h_{j}\right) \cdot[Z]\right)
$$

where $\theta: Z:=Z_{i_{1}, \ldots, i_{k}} \rightarrow X(w)$ is the Bott-Samelson resolution (Lemma 3.1). We have also shown that

$$
c_{\mathrm{SM}}\left(X(w)^{\circ}\right)=\mathcal{T}_{i_{k}} \cdots \mathcal{T}_{i_{1}}([p t])
$$

(Corollary 3.4). Since $c_{\mathrm{SM}}\left(X(w)^{\circ}\right) \in H_{*}(X(w))$, we have

$$
c_{\mathrm{SM}}\left(X(w)^{\circ}\right)=\sum_{u \leqslant w} c(u ; w)[X(u)]
$$

where $c(u ; w)$ are well-defined integers. In fact, $c(w ; w)=1$ since the map $\theta$ is birational, and $c(\mathrm{id} ; w)=1$ since $X(w)^{\circ} \cong \mathbb{A}^{\ell(w)}$ and $\chi\left(\mathbb{A}^{\ell(w)}\right)=1$.

The operator $\mathcal{T}_{k}$ does not preserve positivity: $\mathcal{T}_{k}\left(\left[X\left(s_{k}\right)^{\circ}\right]\right)=-\left[X\left(s_{k}\right)^{\circ}\right]$ by Proposition [4.3, and in fact $\mathcal{T}_{k}\left(\left[X(w)^{\circ}\right]\right)$ may have negative contributions from Schubert classes even if $\ell\left(w s_{k}\right)>\ell(w)$ (Remark 4.5). Examples also show that $c_{\mathrm{SM}}\left(Z^{\circ}\right)$ is not necessarily a positive combination of strata of the normal crossing divisor $D_{Z}:=Z \backslash Z^{\circ}\left(Z_{12321}^{\circ}\right.$ is the smallest such example). So one should not expect any positivity properties of the CSM class a priori. Nevertheless, we conjecture that these classes are positive:

Conjecture 1. For all $u \leqslant w$, the coefficient $c(u ; w)$ from the expansion (15) is strictly positive.

Note that with notation as above, the class of the Schubert variety $X(w)$ is given by

$$
c_{\mathrm{SM}}(X(w))=\sum_{u \leqslant w}\left(\sum_{u \leqslant v \leqslant w} c(u ; v)\right)[X(u)] ;
$$


indeed, $\mathbb{1}_{X(w)}=\sum_{v \leqslant w} \mathbb{1}_{X(v)^{\circ}}$. So Conjecture 1 would imply that these classes are also necessarily effective.

A positivity result analogous to Conjecture 1 was conjectured by the authors in AM09. for Schubert cells in the Grassmannian $\operatorname{Gr}(p, n)$ of subspaces of dimension $p$ in $\mathbb{C}^{n}$. This conjecture was proved in AM09 in the case $p=2$, in Mih15 for $p=3$, and several classes of coefficients were proved to be positive by B. Jones [Jon10] and J. Stryker [Str11]. The full conjecture has recently been proven by June Huh [Huh]. By Proposition 3.5, the CSM classes of Schubert cells in any homogeneous space $G / P$ are in fact push-forwards of CSM classes of Schubert cells in $G / B$; therefore Conjecture 1 would simultaneously imply the positivity of all CSM classes of Schubert cells in all $G / P$, and in particular it would yield an alternative proof of Huh's theorem.

By the same token, Huh's theorem provides some evidence for Conjecture 1, since it implies that $c(u ; w)>0$ in type A when $u$ is a Grassmannian permutation (cf. Example 4.4). In fact, Conjecture 1in type A is also supported by explicit computations of several thousand cases. At the time of this writing, we have verified that the CSM classes of all Schubert cells in $\operatorname{Fl}(n)$ are positive for $n \leqslant 7$ and for all words of length $\leqslant 15$ in $\mathrm{Fl}(8)$.

In the rest of this section we discuss more evidence for Conjecture 1 in all types. We prove positivity in the following cases:

- $c(u ; w)>0$ if $u<w$ and $\ell(w)-\ell(u)=1$ (Corollary [5.2);

- $c(u ; w)>0$ for all $u \leqslant w$ if $w$ admits a decomposition into distinct simple reflections (Corollary 5.4).

These two results will follow from more general considerations which seem independently interesting: the first one is an explicit computation of the codimension 1 term in the CSM class of a Schubert cell (Proposition [5.1), and the second one highlights one case in which the operator $\mathcal{T}_{k}$ does preserve positivity (Proposition 5.3).

Proposition 5.1. Let $\rho=\omega_{1}+\cdots+\omega_{r}$ be the sum of the fundamental weights, and let $w \in W$. Then

$$
c_{S M}\left(X(w)^{\circ}\right)=[X(w)]+c_{1}\left(\mathcal{L}_{\rho}\right) \cdot[X(w)]+\text { lower dimensional terms. }
$$

Proof. Let $w=s_{i_{1}} \cdots s_{i_{k}}$ be a reduced decomposition, and let $Z:=Z_{i_{1}, \ldots, i_{k}}$ with SNC divisor $D_{Z}$, as in 22.3 , By [BK05, Prop. 2.2.2],

$$
K_{Z}=\mathcal{O}_{Z}\left(-D_{Z}\right) \otimes \theta^{*}\left(c_{1}\left(\mathcal{L}_{-\rho}\right)\right)
$$

and hence $c_{1}\left(T_{Z}\right)=\left[D_{Z}\right]+\theta^{*}\left(c_{1}\left(\mathcal{L}_{\rho}\right)\right)$. On the other hand, $c_{1}\left(T_{Z}\right)=\left[D_{Z}\right]+\sum_{i=1}^{k} h_{i}$ by Proposition 2.4 (c). Therefore $h_{1}+\cdots+h_{k}=\theta^{*}\left(c_{1}\left(\mathcal{L}_{\rho}\right)\right)$, and the stated identity follows from (14) and the projection formula.

Corollary 5.2. The coefficient $c(u ; w)>0$ if $u<w$ with $\ell(u)=\ell(w)-1$.

Proof. Recall that $\left\langle\omega_{i}, \alpha_{j}^{\vee}\right\rangle=\delta_{i j}$ (the Kronecker symbol) and in particular $\left\langle\rho, \alpha_{j}^{\vee}\right\rangle>0$ for all simple roots $\alpha_{j}$. Consider $u<w$ such that $\ell(u)=\ell(w)-1$. Then $u=w s_{\beta}$ for some positive root $\beta \in R^{+}$(see e.g. [Hum90, §5.11]). By the Chevalley formula (1), the coefficient of $\left[X\left(w s_{\beta}\right)\right]$ in $c_{1}\left(\mathcal{L}_{\rho}\right) \cap[X(w)]$ equals $\left\langle\rho, \beta^{\vee}\right\rangle>0$, concluding the proof.

Proposition 5.3. Let $w \in W$ be a Weyl group element, and assume $w$ admits a decomposition into simple reflections other than $s_{k}$.

(a) The homology class $T_{k}([X(v)])$ is a non-negative linear combination of Schubert classes $[X(u)]$ with $u \leqslant v s_{k}$. In fact,

$$
\mathcal{T}_{k}([X(v)])=\left[X\left(v s_{k}\right)\right]+[X(v)]+\sum_{u<v s_{k}, u \neq v} d_{k}(u ; v)[X(u)]
$$


with $d_{k}(u ; v) \geqslant 0$ for all $u<v s_{k}$.

(b) Assume in addition that $s_{k}$ commutes with all simple reflections in a decomposition of $v$. Then $d_{k}(u ; v)=0$ for $u<v s_{k}, u \neq v$, that is:

$$
\mathcal{T}_{k}([X(v)])=\left[X\left(v s_{k}\right)\right]+[X(v)] .
$$

Proof. Let $S_{v}:=\left\{s_{i_{1}}, \ldots, s_{i_{t}}\right\}$ be the set of reflections appearing in a reduced decomposition of $v$; this set is independent of the choice of reduced decomposition, since it is preserved by the braid relations in $W$ (see e.g., Hum90, §5.1]). Since every decomposition of $v$ into simple reflections can be reduced to a reduced decomposition, the hypothesis of the proposition implies that $s_{k} \notin S_{v}$ in part (a), and that further $s_{k}$ commutes with all $s_{i_{j}} \in S$ in part (b).

Since $v s_{k}>v$, by Proposition 4.3 we have

$$
\mathcal{T}_{k}([X(v)])=\left[X\left(v s_{k}\right)\right]+[X(v)]+\sum\left\langle\alpha_{k}, \beta^{\vee}\right\rangle\left[X\left(v s_{k} s_{\beta}\right)\right]
$$

where the sum is over all positive $\operatorname{roots} \beta \neq \alpha_{k}$ such that $\ell(v)=\ell\left(v s_{k} s_{\beta}\right)$. We have to prove that, under the hypothesis of the proposition, $\left\langle\alpha_{k}, \beta^{\vee}\right\rangle \geqslant 0$ for all $\beta$ in the range of summation. In fact, all the $\beta$ in this range satisfy $v s_{k} s_{\beta}<v s_{k}$, and we will verify that $\left\langle\alpha_{k}, \beta^{v}\right\rangle \geqslant 0$ for all such reflections $\beta$. By [Hum90, §5.7] the condition $v s_{k}>v$ implies that $v\left(\alpha_{k}\right)>0$, and $v s_{k} s_{\beta}<v s_{k}$ implies that $v s_{k}(\beta)<0$, i.e.,

$$
v\left(\beta-\left\langle\beta, \alpha_{k}^{\vee}\right\rangle \alpha_{k}\right)=v(\beta)-\left\langle\beta, \alpha_{k}^{\vee}\right\rangle v\left(\alpha_{k}\right)<0 .
$$

If $v(\beta)>0$, then we are done, because $v\left(\alpha_{k}\right)>0$. So we assume $v(\beta)<0$, which is equivalent to $v s_{\beta}<v$, and it follows that $v s_{\beta}$ admits a reduced expression only using reflections in $S_{v}$. We deduce that $s_{k}$ does not appear in a reduced expression for $s_{\beta}$, and hence that the simple root $\alpha_{k}$ does not appear in the support of the positive root $\beta$. Since $s_{\alpha_{k}} \notin S_{v}$, it follows that $\alpha_{k}$ does not appear in the support of $v(\beta)$; and $\alpha_{k}$ appears with coefficient +1 in $v\left(\alpha_{k}\right)$. Then (18) forces $\left\langle\beta, \alpha_{k}^{\vee}\right\rangle \geqslant 0$ as claimed. This proves part (a).

To prove part (b) we use a similar argument. By (17), it suffices to show that $\left\langle\alpha_{k}, \beta^{\vee}\right\rangle=0$ for all reflections $s_{\beta}$ such that $v s_{k} s_{\beta}<v s_{k}$. This relation implies that $s_{\beta}$ has a reduced decomposition containing only simple reflections in an expression for $v s_{k}$. But $\beta \neq \alpha_{k}$, and no simple reflections $s_{\alpha_{j}}$ with $\alpha_{j}$ adjacent to $\alpha_{k}$ in the Dynkin diagram for $G$ can appear in the decomposition of $s_{\beta}$ : otherwise such reflections would appear in $S_{v}$, contradicting the commutativity hypothesis. This implies that the support of $\beta$ does not contain any simple root adjacent to $\alpha_{k}$, thus $\left\langle\alpha_{k}, \beta^{\vee}\right\rangle=0$, concluding the proof.

Corollary 5.4. Let $w \in W$ be a Weyl group element, and assume $w$ admits a decomposition into simple reflections other than $s_{k}$. If $c(v ; w)>0$ for all $v \leqslant w$, then $c\left(u ; w s_{k}\right)>0$ for all $u \leqslant w s_{k}$.

In particular, if $w \in W$ admits a decomposition into distinct simple reflections, then $c(u ; w)>0$ for all $u \leqslant w$.

Proof. The second statement follows from the first by an immediate induction. To prove the first statement, note that if $s_{k}$ does not appear in a decomposition for $w$, then it does not appear in a reduced decomposition for $w$, and hence it does not appear in a decomposition for $v$. Thus the hypothesis of Proposition 5.3 applies to all $v \leqslant w$. By Corollary 3.4 we have

$$
\begin{aligned}
c_{\mathrm{SM}}\left(X\left(w s_{k}\right)^{\circ}\right) & =\mathcal{T}_{k}\left(c_{\mathrm{SM}}(w)^{\circ}\right)=\mathcal{T}_{k} \sum_{v \leqslant w} c(v ; w)[X(v)] \\
& =\sum_{v \leqslant w} c(v ; w)\left(\left[X\left(v s_{k}\right)\right]+[X(v)]+\sum_{u^{\prime}<v s_{k}, u^{\prime} \neq v} d_{k}\left(u^{\prime} ; v\right)\left[X\left(u^{\prime}\right)\right]\right)
\end{aligned}
$$


with $c(v ; w)>0$ by hypothesis and $d_{k}\left(u^{\prime} ; v\right) \geqslant 0$ by Proposition 5.3. The statement is immediate from this expression, since $u \leqslant w s_{k}$ implies that $u$ has a reduced expression which is a subexpression of one for $w s_{k}$, thus either $u=v \leqslant w$ or $u=v s_{k}$ with $v \leqslant w$.

A particular case of Corollary 5.4 is particularly vivid: if $w=s_{i_{1}} \cdots s_{i_{k}}$ is a reduced decomposition and the simple reflections $s_{i_{1}}, \ldots, s_{i_{k}}$ commute with one another, then an induction argument based on Proposition 5.3(b) implies that

$$
c_{\mathrm{SM}}\left(X(w)^{\circ}\right)=\sum_{u \leqslant w}[X(u)]
$$

Notice that if $w$ satisfies this condition, then so does every $v$ preceding it in the Bruhat order. Then (16) and (19) give the CSM class of the Schubert variety

$$
c_{\mathrm{SM}}(X(w))=\sum_{u \leqslant w} 2^{\ell(w)-\ell(u)}[X(u)],
$$

for every $w \in W$ decomposing into commuting simple reflections.

\section{Equivariant Chern-Schwartz-MacPherson classes of Schubert cells}

In this section we extend our calculation of CSM classes to the T-equivariant situation. We will show that the same difference $\partial_{k}-s_{k}$ defines an operator $\mathcal{T}_{k}^{T}$ on equivariant homology $H_{*}^{T}(G / B)$, sending an (equivariant) CSM class $c_{\mathrm{SM}}^{T}\left(X(w)^{\circ}\right)$ to the class $c_{\mathrm{SM}}{ }^{T}\left(X\left(w s_{k}\right)^{\circ}\right)$. The proof of Lemma2.2 extends to the equivariant setting, and shows that $\left(\partial_{k}-s_{k}\right)^{2}=$ id. In particular, the operators $\mathcal{T}_{k}^{T}$ give a representation of $W$ on equivariant homology. The proof that $\mathcal{T}_{k}^{T}$ acts as expected on CSM classes is essentially identical to the proof in the non-equivariant case; we only need to verify that no 'equivariant corrections' are introduced in the recursion formula from Corollary 3.4 .

6.1. Equivariant CSM classes. Recall that $T \subset B$ is the maximal torus in the Borel subgroup $B$. If $X$ is a variety with a $T$-action, then the equivariant cohomology $H_{T}^{*}(X)$ is the ordinary cohomology of the Borel mixing space $X_{T}:=(E T \times X) / T$, where $E T$ is the universal $T$-bundle and $T$ acts by $t \cdot(e, x)=\left(e t^{-1}, t x\right)$. It is an algebra over $H_{T}^{*}(p t)$, a polynomial ring $\mathbb{Z}\left[t_{1}, \ldots, t_{r}\right]$, where $t_{i}, \ldots, t_{r}$ form generators for the weight lattice of $T$. We address the reader to $\mathrm{Knu}$ or $\mathrm{Ohm} 06$ for basic facts on equivariant cohomology. Since $X$ is smooth, we can and will identify the equivariant homology $H_{*}^{T}(X)$ with the equivariant cohomology $H_{T}^{*}(X)$. Every closed subvariety $Y \subseteq X$ that is invariant under the $T$ action determines an equivariant fundamental class $[Y]_{T} \in H_{*}^{T}(X)$.

Ohmoto defines the group of equivariant constructible functions $\mathcal{F}^{T}(X)$ (for tori and for more general groups) in [Ohm06, §2]. We recall the main properties that we need:

(1) If $W \subset X$ is a constructible set which is invariant under the $T$-action, its characteristic function $\mathbb{1}_{W}$ is an element of $\mathcal{F}^{T}(X)$. (The group $\mathcal{F}^{T}(X)$ also contains other elements, but this will be immaterial for us.)

(2) Every proper $T$-equivariant morphism $f: Y \rightarrow X$ of algebraic varieties induces a homomorphism $f_{*}^{T}: \mathcal{F}^{T}(X) \rightarrow \mathcal{F}^{T}(Y)$. The restriction of $f_{*}^{T}$ to characteristic functions of constructible $T$-invariant sets coincides with the ordinary push-forward $f_{*}$ of constructible functions. See [Ohm06, §2.6].

Ohmoto proves Ohm06, Theorem 1.1] that there is an equivariant version of MacPherson transformation $c_{*}^{T}: \mathcal{F}^{T}(X) \rightarrow H_{*}^{T}(X)$ that satisfies $c_{*}^{T}\left(\mathbb{1}_{Y}\right)=c^{T}\left(T_{Y}\right) \cap[Y]_{T}$ if $Y$ is a projective, non-singular variety, and that is functorial with respect to proper push-forwards. 
The last statement means that for all proper $T$-equivariant morphisms $Y \rightarrow X$ the following diagram commutes:

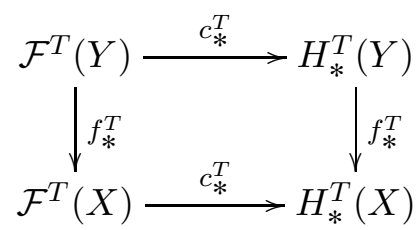

We denote by $c_{\mathrm{SM}}{ }^{T}\left(X(w)^{\circ}\right):=c_{*}^{T}\left(\mathbb{1}_{X(w)^{\circ}}\right)$ the equivariant CSM class of the Schubert cell corresponding to an element $w \in W$.

Finally, we note that both the BGG operator $\partial_{i}$ and the right Weyl group action $s_{i}$ are induced by morphisms which commute with the $T$-action. It follows that they both determine $H_{T}^{*}(p t)$-algebra endomorphisms of $H_{T}^{*}(G / B)$, for which we will use the same notation. Further, Proposition 2.1 and formula (3) extend to the equivariant setting after replacing the Chern class $c_{1}\left(\mathcal{L}_{\alpha_{k}}\right)$ by its equivariant version $c_{1}^{T}\left(\mathcal{L}_{\alpha_{k}}\right)$. We refer to [Knu, $\left.\S 3\right]$ for details. The analogue of identity (4) can be found in [Knu, $\S 4$, Corollary]; the equivariant version includes additional terms.

6.2. Equivariant CSM classes via the operator $\mathcal{T}_{k}^{T}=\partial_{k}-s_{k}$. In this section we give the proof of the equivariant version of Theorem 1.1.

Recall the diagram (5) from 2.3 .

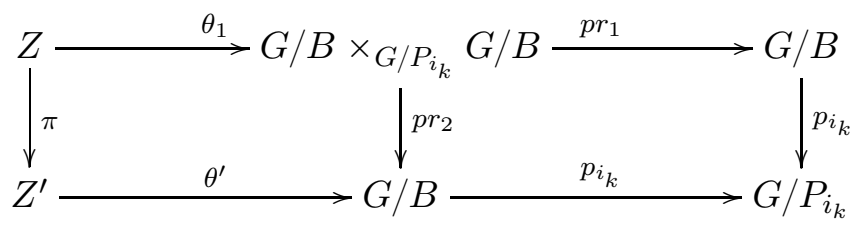

Recall that $Z^{\prime}$ is the Bott-Samelson variety for a Weyl group element $w^{\prime} \in W$ and that $Z$ is the Bott-Samelson variety corresponding to $w^{\prime} s_{i_{k}}$, where $\ell\left(w^{\prime} s_{i_{k}}\right)>\ell\left(w^{\prime}\right)$.

Recall also that $G / B$ is a $\mathbb{P}^{1}$-bundle $\mathbb{P}(E)$ for some rank-2, equivariant vector bundle over $G / P_{i_{k}}$, and that we defined $\mathcal{E}:=E \otimes \mathcal{O}_{E}(1)$, a vector bundle over $G / B$. This bundle fits into the exact sequence ([6) of equivariant vector bundles

$$
0 \longrightarrow \mathcal{O} \longrightarrow \mathcal{E} \longrightarrow \mathcal{Q} \longrightarrow 0
$$

where the action on $\mathcal{O}$ is trivial, and $\mathcal{Q}=T_{p_{i_{k}}}$. The inclusion $\mathcal{O} \subset \mathcal{E}$ determines an equivariant section $\sigma_{i_{k}}: G / B \rightarrow \mathbb{P}(\mathcal{E})=G / B \times_{G / P_{i_{k}}} G / B$, inducing the section $\sigma$ of $\pi$ introduced in 2.3 .

Lemma 6.1. The image of the section $\sigma_{i_{k}}$ is the diagonal $\Delta \subset G / B \times{ }_{G / P_{i_{k}}} G / B$.

Proof. The image of $\sigma_{i_{k}}$ is $\mathbb{P}(\mathcal{O})$, which maps identically to $G / B$ via both $p r_{1}$ and $p r_{2}$.

Proposition 6.2. $\quad$ (a) We have an isomorphism of equivariant bundles

$$
\mathcal{O}_{\mathcal{E}}(1)=p r_{2}^{*} \mathcal{O}_{E}(-1) \otimes p r_{1}^{*} \mathcal{O}_{E}(1) \text {. }
$$

(b) The diagonal $\Delta$ is the zero locus of a homogeneous section of the bundle

$$
\mathcal{O}_{G / B \times \times_{G / P_{i_{k}}} G / B}(\Delta)=\operatorname{pr}_{2}^{*}(\mathcal{Q}) \otimes \mathcal{O}_{\mathcal{E}}(1) .
$$

(c) Let $[\Delta]_{T} \in H_{*}^{T}\left(G / B \times_{G / P_{i_{k}}} G / B\right)$ be the equivariant fundamental class determined by $\Delta$. Then

$$
[\Delta]_{T}=c_{1}^{T}\left(\mathcal{O}_{G / B \times \times_{G / P_{k}}} G / B(\Delta)\right) \cap\left[G / B \times_{G / P_{i_{k}}} G / B\right]_{T} .
$$


Proof. Part (a) was proved within the proof of Theorem 3.3. The inclusion $\Delta \subset G / B \times G / P_{i_{k}}$ $G / B$ is given by $\mathbb{P}(\mathcal{O}) \subset \mathbb{P}(\mathcal{E})$. Then by [Ful98, Appendix B.5.6] $\Delta$ is the zero locus of a section of the bundle $\mathcal{Q} \otimes \mathcal{O}_{\mathcal{E}}(1)$. This establishes (b).

Part (c) follows from a general fact: if an equivariant divisor $D$ is the zero scheme of a homogeneous section of an equivariant line bundle $\mathcal{L}$ on $G / B$, then $[D]_{T}=c_{1}^{T}(\mathcal{L}) \cap$ $[G / B]_{T}$. This follows from the analogous non-equivariant statement in the corresponding Borel mixing space.

The next observation is that (9) extends to the equivariant case.

Let $Z$ be a variety with a $T$-action, and let $D \subseteq Z$ be a divisor with simple normal crossings and equivariant components $D_{i}$. Then

$$
c_{\mathrm{SM}}^{T}(Z \backslash D)=\frac{c^{T}\left(T_{Z}\right)}{\prod_{j}\left(1+D_{i}^{T}\right)} \cap[Z]_{T},
$$

where $D_{i}^{T}=c_{1}^{T}\left(\mathcal{O}\left(D_{i}\right)\right)$ (so that $\left.D_{i}^{T} \cap[Z]_{T}=\left[D_{i}\right]_{T}\right)$.

This may be proven by the same method used in the proof of [Alu99, Theorem 1].

Now let $Z=Z_{i_{1}, \ldots, i_{k}}$ and let $D=D_{Z}$ be the SNC divisor defined in 92.3 . Recall that $D=\pi^{-1}\left(D_{Z^{\prime}}\right) \cup D_{k}$. The following is the equivariant analogue of Lemma 3.2.

Lemma 6.3. The following identity holds in $H_{*}^{T}(G / B)$ :

$$
c_{S M}^{T}\left(X(w)^{\circ}\right)=\theta_{*}\left(\left(1+h_{k}^{T}\right) \cdot \pi^{*}\left(c_{S M}^{T}\left(Z^{\prime \circ}\right)\right)\right)
$$

where $h_{k}^{T}=\left(\theta^{\prime}\right)^{*} c_{1}^{T}\left(\mathcal{O}_{\mathcal{E}}(1)\right)$.

Proof. By Proposition $6.2(\mathrm{~b}), \Delta^{T}=c_{1}^{T}\left(p r_{2}^{*}(\mathcal{Q}) \otimes \mathcal{O}_{\mathcal{E}}(1)\right)$. Pulling back by $\theta^{\prime}$, we obtain $D_{k}^{T}=c_{1}^{T}\left(\pi^{*}\left(\theta^{\prime *} \mathcal{Q}\right) \otimes \mathcal{O}_{\mathcal{E}}(1)\right)$, and this implies

$$
c^{T}\left(T_{Z}\right)=\pi^{*}\left(c^{T}\left(T_{Z^{\prime}}\right)\right)\left(1+h_{k}^{T}\right)\left(1+D_{k}^{T}\right),
$$

arguing exactly as in the proof of Proposition 2.4 (c). The stated identity follows then from (21) by the same argument proving Lemma 3.2 from (91).

Theorem 6.4. Let $\mathcal{T}_{k}^{T}: H_{T}^{*}(G / B) \rightarrow H_{T}^{*}(G / B)$ be the operator

$$
\mathcal{T}_{k}^{T}=\left(1+c_{1}^{T}\left(\mathcal{L}_{-\alpha_{k}}\right)\right) \partial_{k}-i d=\partial_{k}-s_{k} .
$$

Then $\mathcal{T}_{k}^{T}\left(c_{S M}^{T}\left(X(w)^{\circ}\right)\right)=c_{S M}^{T}\left(X\left(w s_{k}\right)^{\circ}\right)$.

Proof. The same proof applies as in the non-equivariant case, taking into account that all maps used are $T$-equivariant and that the statement of Proposition 2.1 extends without changes to the equivariant setting.

The equivariant versions of the identities (2) and (4) yield the following explicit formula for the equivariant operator $\mathcal{T}_{k}^{T}$ :

\section{Proposition 6.5.}

$\mathcal{T}_{k}^{T}([X(w)])= \begin{cases}-[X(w)] & \text { if } \ell\left(w s_{k}\right)<\ell(w) \\ \left(1+w\left(\alpha_{k}\right)\right)\left[X\left(w s_{k}\right)\right]+[X(w)]+\Sigma\left\langle\alpha_{k}, \beta^{\vee}\right\rangle\left[X\left(w s_{k} s_{\beta}\right)\right] & \text { if } \ell\left(w s_{k}\right)>\ell(w)\end{cases}$ where the sum is over all positive roots $\beta \neq \alpha_{k}$ such that $\ell(w)=\ell\left(w s_{k} s_{\beta}\right)$, and $w\left(\alpha_{k}\right)$ denotes the natural $W$ action on roots.

Notice that $w s_{k}>w$ implies that $w\left(\alpha_{k}\right)>0$. 
6.3. Equivariant positivity. Theorem 6.4 and Proposition 6.5 give an effective way to compute equivariant Chern-Schwartz-MacPherson classes of Schubert cells in $G / B$. Since the equivariant classes $[X(u)]^{T}$ form a basis of $H_{*}^{T}(G / B)$ over $H_{*}^{T}(p t)=\mathbb{Z}\left[t_{1}, \ldots, t_{r}\right]$, we have an equivariant analogue of (15):

$$
c_{\mathrm{SM}}^{T}\left(X(w)^{\circ}\right)=\sum_{u \leqslant w} c^{T}(u ; w)[X(u)]_{T}
$$

where $c^{T}(u ; w)$ are well-defined polynomials in the $t_{i}$ 's.

Remark 6.6. • Proposition 6.5 implies that $c^{T}(u ; w)$ is a polynomial with integer coefficients in the roots $\alpha_{i}$; for example, $c^{T}(u ; w)$ is a polynomial in $t_{i}-t_{i+1}$ in type A.

- The constant term in $c^{T}(u ; w)$ equals the non-equivariant coefficient $c(u ; w)$.

- By general considerations (see e.g., $\mathrm{Ohm06}, \S 4.1]), c_{\mathrm{SM}}^{T}\left(X(w)^{\circ}\right)$ has no nonzero components in $H_{i}^{T}(G / B)$ for $i<0$. This implies that the polynomials $c^{T}(u ; w)$ have degree at $\operatorname{most} \operatorname{dim}(X(u))=\ell(u)$ in the roots $\alpha_{i}$. In particular $c^{T}$ (id; $\left.w\right)$ is constant, and it follows that $c^{T}(\mathrm{id} ; w)=1$ for all $w$.

- The sum $\sum_{u, w \in W} c^{T}(u ; w)[X(w)]^{T}$ equals the total equivariant Chern class of the flag manifold $G / B$. Now, we have the identity

$$
c^{T}\left(T_{G / B}\right)=\prod_{\alpha \in R^{+}}\left(1+c_{1}^{T}\left(\mathcal{L}_{\alpha}\right)\right)
$$

where $\mathcal{L}_{\alpha}$ is the line bundle defined in 92.1 . Indeed, $T_{G / B}$ is the homogeneous bundle $G \times{ }^{B}(\operatorname{Lie}(G) / \operatorname{Lie}(B))=G \times^{B} \operatorname{Lie}\left(U^{-}\right)$where $U^{-}$is the opposite unipotent group; it follows that the weights at the $B$-fixed point are the negative roots of $(G, B)$. In particular, the localization at id. $B$ of $c^{T}\left(T_{G / B}\right)$ equals

$$
\iota_{\mathrm{id} . B}^{*} c^{T}\left(T_{G / B}\right)=\prod_{\alpha \in R^{+}}(1-\alpha)=\prod_{\alpha \in R^{+}} \iota_{\mathrm{id} . B}^{*} c^{T}\left(\mathcal{L}_{\alpha}\right) .
$$

The stated identity follows by homogeneity. Since the polynomial $c^{T}\left(w_{0} ; w_{0}\right)$ equals the coefficient of $[G / B]_{T}$ in this class, we must have

$$
c^{T}\left(w_{0} ; w_{0}\right)=\prod_{\alpha \in R^{+}}(1+\alpha)
$$

where the product ranges over all positive roots.

- More generally, let $w=s_{i_{1}} \ldots s_{i_{k}}$ be a reduced decomposition. It follows from Proposition 6.5 that the leading term of $c_{\mathrm{SM}^{T}}\left(X(w)^{\circ}\right)=\left(T_{i_{k}} \ldots T_{i_{1}}\right)[X(i d)]_{T}$ is

$$
c^{T}(w ; w)=\prod_{t=1}^{k}\left(1+s_{i_{1}} \ldots s_{i_{t-1}}\left(\alpha_{i_{t}}\right)\right),
$$

with the convention that $s_{i_{0}}=i d$. If $w=w_{0}$, then the set of roots $s_{i_{1}} \ldots s_{i_{t-1}}\left(\alpha_{i_{t}}\right)$ obtained as $t$ varies coincides with the set of positive roots, and one recovers (23).

The following statement generalizes Conjecture 1,

Conjecture 2. For all $u \leqslant w$, the coefficient $c^{T}(u ; w)$ is a polynomial with positive coefficients in the simple roots $\alpha_{i}$.

This statement is supported by explicit computations for low dimensions in type A. 
Example 6.7. Let $\Gamma_{T}$ be the matrix whose $(u, w)$ entry is $c^{T}(u ; w)$. For $F l(3)$, listing the elements of $S_{3}$ in the order (123), (132), (213), (231), (312), (321), we have

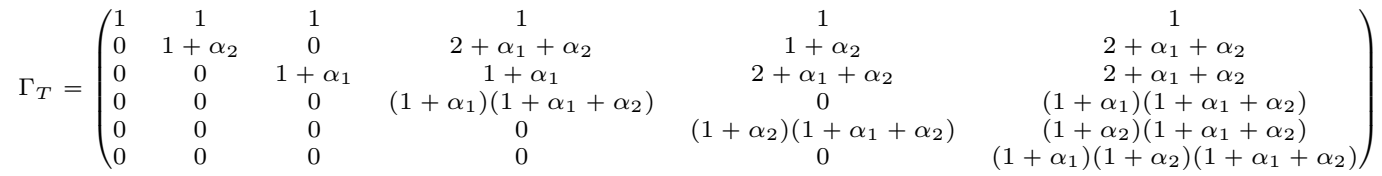

verifying Conjecture 2 in this case.

\section{REFERENCES}

[Alu99] Paolo Aluffi. Differential forms with logarithmic poles and Chern-Schwartz-MacPherson classes of singular varieties. C. R. Acad. Sci. Paris Sér. I Math., 329(7):619-624, 1999.

[Alu06a] Paolo Aluffi. Classes de Chern des variétés singulières, revisitées. C. R. Math. Acad. Sci. Paris, 342(6):405-410, 2006.

[Alu06b] Paolo Aluffi. Limits of Chow groups, and a new construction of Chern-Schwartz-MacPherson classes. Pure Appl. Math. Q., 2(4):915-941, 2006.

[AM09] Paolo Aluffi and Leonardo Constantin Mihalcea. Chern classes of Schubert cells and varieties. J. Algebraic Geom., 18(1):63-100, 2009.

[BGG73] I. N. Bernšteřn, I. M. Gel'fand, and S. I. Gel'fand. Schubert cells, and the cohomology of the spaces G/P. Uspehi Mat. Nauk, 28(3(171)):3-26, 1973.

[BK05] Michel Brion and Shrawan Kumar. Frobenius splitting methods in geometry and representation theory, volume 231 of Progress in Mathematics. Birkhäuser Boston, Inc., Boston, MA, 2005.

[BL00] Sara Billey and V. Lakshmibai. Singular loci of Schubert varieties, volume 182 of Progress in Mathematics. Birkhäuser Boston, Inc., Boston, MA, 2000.

[Ful98] William Fulton. Intersection theory, volume 2 of Ergebnisse der Mathematik und ihrer Grenzgebiete. 3. Folge. A Series of Modern Surveys in Mathematics [Results in Mathematics and Related Areas. 3rd Series. A Series of Modern Surveys in Mathematics]. Springer-Verlag, Berlin, second edition, 1998.

[FW04] W. Fulton and C. Woodward. On the quantum product of Schubert classes. J. Algebraic Geom., 13(4):641-661, 2004.

[Gin86] V. Ginzburg. Characteristic varieties and vanishing cycles. Inv. Math., 84:327-402, 1986.

[Gin98] Victor Ginzburg. Geometric methods in the representation theory of Hecke algebras and quantum groups. In Representation theories and algebraic geometry (Montreal, PQ, 1997), volume 514 of NATO Adv. Sci. Inst. Ser. C Math. Phys. Sci., pages 127-183. Kluwer Acad. Publ., Dordrecht, 1998. Notes by Vladimir Baranovsky [V. Yu. Baranovski1].

[GP02] Mark Goresky and William Pardon. Chern classes of automorphic vector bundles. Invent. Math., 147(3):561-612, 2002.

[Huh] June Huh. Positivity of Chern classes of Schubert cells and varieties. to appear in J. of Algebraic Geometry, available at: ar $\chi$ iv: 1302.5852 .

[Hum90] James E. Humphreys. Reflection groups and Coxeter groups, volume 29 of Cambridge Studies in Advanced Mathematics. Cambridge University Press, Cambridge, 1990.

[Jon10] Benjamin F. Jones. Singular Chern classes of Schubert varieties via small resolution. Int. Math. Res. Not. IMRN, (8):1371-1416, 2010.

[Ken90] Gary Kennedy. MacPherson's Chern classes of singular algebraic varieties. Comm. Algebra, 18(9):2821-2839, 1990.

[Knu] A. Knutson. A Schubert Calculus recurrence from the noncomplex $W$-action on $G / B$. arXiv.math/0306304v1.

[Mac74] R. D. MacPherson. Chern classes for singular algebraic varieties. Ann. of Math. (2), 100:423-432, 1974 .

[Mih15] Leonardo Constantin Mihalcea. Binomial determinants and positivity of Chern-SchwartzMacPherson classes. Australasian Journal of Combinatorics., 62(2):155-171, 2015.

[MO] D. Maulik and A. Okounkov. Quantum groups and quantum cohomology. arXiv:1211.1287.

[Ohm06] Toru Ohmoto. Equivariant Chern classes of singular algebraic varieties with group actions. Math. Proc. Cambridge Philos. Soc., 140(1):115-134, 2006.

[RTV14] R. Rimányi, V. Tarasov, and A. Varchenko. Cohomology classes of conormal bundles of Schubert varieties and Yangian weight functions. Math. Z., 277(3-4):1085-1104, 2014. 
[RV] R. Rimányi and A. Varchenko. Equivariant Chern-Schwartz-MacPherson classes in partial flag varieties: interpolation and formulae. arXiv:1509.09315.

[Sch65a] Marie-Hélène Schwartz. Classes caractéristiques définies par une stratification d'une variété analytique complexe. I. C. R. Acad. Sci. Paris, 260:3262-3264, 1965.

[Sch65b] Marie-Hélène Schwartz. Classes caractéristiques définies par une stratification d'une variété analytique complexe. II. C. R. Acad. Sci. Paris, 260:3535-3537, 1965.

[Str11] Judson P. III Stryker. Chern-Schwartz-MacPherson classes of graph hypersurfaces and Schubert varieties, 2011. Thesis (Ph.D.) - The Florida State University.

[Su] C. Su. Restriction formula for stable basis of the Springer resolution. arXiv:1501.04214.

[Tym08] Julianna S. Tymoczko. Permutation actions on equivariant cohomology of flag varieties. In Toric topology, volume 460 of Contemp. Math., pages 365-384. Amer. Math. Soc., Providence, RI, 2008.

[Web12] Andrzej Weber. Equivariant Chern classes and localization theorem. J. Singul., 5:153-176, 2012.

Mathematics Department, Florida State University, Tallahassee FL 32306

E-mail address: aluffi@math.fsu.edu

Department of Mathematics, Virginia Tech University, Blacksburg, VA 24061

E-mail address: lmihalce@vt.edu 